\title{
Lower levels of serum albumin are associated with impairment of cognitive function in cirrhotic patients with early-stage hepatic encephalopathy: An exploratory data analysis of phase II/III clinical trials of rifaximin in Japan
}

Kosuke Kaji ( $\sim$ kajik@naramed-u.ac.jp )

Nara Medical University

Kiwamu Okita

Shunan Memorial Hospital

Kazuyuki Suzuki

Iwate Medical University

Ikuya Sato

ASKA Pharmaceutical Co., Ltd.

Masaki Fujisawa

ASKA Pharmaceutical Co., Ltd.

Hitoshi Yoshiji

Department of Gastroenterology, Nara Medical University

Research article

Keywords: Liver cirrhosis, Hepatic encephalopathy, Albumin

Posted Date: March 18th, 2020

DOI: https://doi.org/10.21203/rs.3.rs-17636/v1

License: (c) (i) This work is licensed under a Creative Commons Attribution 4.0 International License.

Read Full License 


\section{Abstract}

Background Because covert hepatic encephalopathy (CHE) has been shown to affect the prognosis of cirrhotic patients, early diagnosis of HE is a prerequisite for preservation of patients' quality of life and for prophylaxis of overt HE. Currently, neuropsychological tests are used for the diagnosis of early-stage HE including $\mathrm{CHE}$. However, it would be inefficient to apply them to all cirrhotic patients. Thus, some biomarkers correlated with the above diagnostic modalities are available for screening examination. The aim of this study was to identify a clinical parameter to predict impairment of cognitive function in cirrhotic patients with early-stage HE.

Methods This exploratory data analysis was based on the data from 172 patients with cirrhotic or idiopathic portosystemic shunt (PSS) in phase II/III trials of rifaximin in Japan. Their data at baseline before treatment with rifaximin were utilized to analyze the relationship between cognitive dysfunction and different clinical parameters We Classification and regression trees (CART) were constructed to identify clinical profiles related to cognitive dysfunction, as indicated by the prolongation of time required for the number connection test (NCT-B).

Results CART analysis detected age 65 years as the variable for the initial split, and serum albumin level was selected as the variable for the second split among patients aged $\leq 65$ years. In 27 cirrhotic patients aged $\leq 65$ years without PSS, receiver-operating characteristic curve analysis revealed that the optimal albumin level cutoff point was $3.05 \mathrm{~g} / \mathrm{dL}$, and the area under the curve was 0.80 for the prolongation of NCT-B time, which was higher than that of other HE-related parameters including the branched-chain amino acids-to-tyrosine ratio (0.46), the prothrombin time-international normalized ratio (PT-INR) (0.68), serum ammonia (0.61), and total bilirubin (0.69).

Conclusions Lower serum albumin level as a clinical biomarker associated with impaired cognitive function is available as a screening examination for early-stage HE in cirrhotic patients aged $\leq 65$ years without PSS before undergoing neuropsychological tests.

\section{Background}

Hepatic encephalopathy (HE) is a significant complication of severe acute or chronic hepatic insufficiency, which is characterized by a wide range of changes in mental state from minimal signs of altered brain function to deep coma [1, 2]. HE is caused by a combination of distinct pathophysiological mechanisms, including inflammation, oxidative stress, increased blood-brain barrier permeability, and energy metabolism, as well as putative neurotoxins, including ammonia, short-chain fatty acids, mercaptans, false neurotransmitters (e.g., tyramine, octopamine, and beta-phenylethanolamines), manganese, and $y$-aminobutyric acid (GABA) - though ammonia [1-3].

Currently, covert $\mathrm{HE}$ (CHE), the earlier stage of $\mathrm{HE}$, is attracting attention. CHE impairs performance on psychometric tests, such as working memory, psychomotor speed, and visuospatial ability, as well as electrophysiological and other functional brain measurements, without any apparent clinical 
manifestations $[4,5]$. These cognitive dysfunction and subclinical symptoms frequently precede further symptoms of $\mathrm{HE}$ and cause inconvenience to the patient's daily life. For example, patients with $\mathrm{CHE}$ often have sleep disorders and deficits in specific activities, such as driving, which are a danger to themselves and others [4-6]. Therefore, early diagnosis and treatment of $\mathrm{CHE}$ is a prerequisite for preservation of the quality of life and for prophylaxis of overt HE in cirrhotic patients.

A full overview of the different diagnostic modalities of $\mathrm{CHE}$ has recently been published. According to the literature, tests used for diagnosing CHE include the Number Connection Tests (NCT) A and B, the Digit Symbol Test, the Block Design Test, the portosystemic encephalopathy (PSE) Syndrome Testproviding the Psychometric Hepatic Encephalopathy Score, the Inhibitory Control Test, the Stroop Test, the Scan Test, the Continuous Reaction Time Test, the Cognitive Drug Research test battery, or the Repeatable Battery for the Assessment of Neuropsychological Status [7-12]. Although these tests are noninvasive and feasible for patients in clinical practice, it would be inefficient to apply them to all cirrhotic patients. Moreover, these tests are incapable of discrimination between cirrhosis-based cognitive dysfunction and senility or dementia. Thus, some biomarkers correlated with the above diagnostic modalities are available for screening examination.

To identify a candidate as a biomarker, this study employed exploratory data analysis (EDA), a statistical assessment to extract meaningful hypotheses or productive knowledge by using a graphical approach $[13,14]$. Among various tools for EDA, the classification and regression tree (CART) is available as a statistical method that uses a constructed model to repeatedly recursively divide the explanatory variable involved in the response $[15,16]$. The factorial structure of the underlying data is visually represented by a "tree." Although EDA is useful for identification of biomarkers, it has never been used to investigate associations between serum parameters and neuropsychological functions in patients with HE.

The aim of this study was to identify a clinical parameter to predict the prolongation of time required for the number connection test (NCT-B) in patients with early-stage HE.

\section{Methods}

\section{Study design and ethical approval}

This study was undertaken as a stratified analysis of phase II/III clinical trials of rifaximin to evaluate the relationship between cirrhosis-related clinical parameters and neuropsychological function and identify an available biomarker to predict the presence of early-stage HE [17]. The study protocols conformed to the principles outlined in the 1964 Declaration of Helsinki and its later amendments and were approved by each institution's review board.

\section{Subjects}

The subjects of this study were 172 patients from the above clinical trials with cirrhotic or idiopathic portosystemic shunt (PSS). They were diagnosed as early-stage HE (grade I or II HE) according to the 
criteria of the Inuyama Symposium in Japan and the presence of hyperammonemia (serum ammonia level $\geq 80 \mu \mathrm{g} / \mathrm{dL}$ ) [18]. The subjects were limited to patients who were between 20 and 74 years of age at the time of informed consent. The major exclusion criteria were psychiatric comorbidities, any comorbidity or medical history found by the investigator to affect evaluation, acute hepatitis, acute liver failure, acute exacerbation of chronic hepatitis, and laboratory data showing total bilirubin $\geq 5.0 \mathrm{mg} / \mathrm{dL}$, hemoglobin $\leq 8 \mathrm{~g} / \mathrm{dL}$, serum potassium $\leq 2.5 \mathrm{mEq} / \mathrm{L}$, or both blood urea nitrogen $\geq 25 \mathrm{mg} / \mathrm{dL}$ and serum creatinine $\geq 2.0 \mathrm{mg} / \mathrm{dL}$.

\section{Data collection}

The following variables were recorded: age; sex; body weight; white blood cell count; platelet count; prothrombin time-international normalized ratio (PT-INR); serum levels of aspartate aminotransferase (AST), alanine aminotransferase, alkaline phosphatase, $\mathrm{y}$-glutamyl transpeptidase, total protein, albumin, total bilirubin, and creatinine; and branched-chain amino acids (BCAA)-to-tyrosine ratio (BTR). The presence of portal systemic shunt, esophageal varix, and ascites was evaluated by abdominal images, including ultrasonography, computed tomography, magnetic resonance imaging, and endoscopy. The PSE index was calculated using the following formula, as previously described: PSE index $=(3 \times \mathrm{HE}$ coma score + blood ammonia score + asterixis score + number connection test A [NCT-A] score)/24 [17].

\section{Neuropsychological functions}

Neuropsychological functions were assessed by the NCT-B using neuropsychological test system software. The software was distributed by the Japan Society of Hepatology [19]. The hardware consisted of a touch screen tablet, such as an iPad (Apple, Cupertino, CA, USA).

\section{Classification and regression trees}

CART were constructed to identify the clinical profiles related to the time required for the NCT-B, as previously described $[15,16]$. The tree plot was visualized with a regression tree model for the NCT-B, and the box plot shows the distribution of observations for nodes and leaves. The preconditions for splitting with CART were as follows: the complexity parameter was set to 0.02 , with 40 observations as the minimum number per node.

\section{Receiver-operating characteristic curve analysis}

Receiver-operating characteristic (ROC) curve analysis was performed to validate the predictive performance of the clinical profiles in identifying the impairment of NCT-B time. The amount of prolongation of NCT-B time in the patients differed according to age: age $<45,51.1 \mathrm{sec} ; 45 \leq$ age $<50$, $50.2 \mathrm{sec} ; 50 \leq$ age < 55, $51.2 \mathrm{sec} ; 55 \leq$ age < 60, $62.2 \mathrm{sec} ; 60 \leq$ age $<65,71.3 \mathrm{sec}$; and $65 \leq$ age $<70$, $87.2 \mathrm{sec}$ [19]. ROC curve analysis obtained the optimal clinical profiles cutoff point. Evaluation indices of the ROC curve were calculated for sensitivity, specificity, positive predictive value (PPV), negative predictive value (NPV), and accuracy on the cutoff point. In addition, the variability of the area under the receiver-operating characteristic (AUROC) curves was assessed by using 2000 stratified bootstrap 
replicates. The summary statistics of AUROC showed the median and two-sided $95 \%$ confidence intervals (Cls).

\section{Statistics}

The data were expressed as numbers, means \pm standard deviation, and medians (range). Differences between groups were estimated using their mean difference and two-sided $95 \%$ Cls. Statistical analyses were performed using R software (http://www.Rproject. org/) as previously described [20]. Associations between clinical parameters and neuropsychological function were evaluated by EDA.

\section{Results}

\section{CARTs for clinical profiles associated with the time required for the NCT-B}

Among the total of 172 patients with early-stage HE, the mean time required for the NCT-B (NCT-B time) was $108.0 \pm 48.1 \mathrm{sec}$. Age was selected as the variable for the initial split, and NCT-B time was $87.1 \pm$ $41.0 \mathrm{sec}$ in patients aged $\leq 65$ years (Figure 1). In 91 patients aged $\leq 65$ years, serum albumin level was selected as the variable for the second split; the NCT-B time was $108.5 \pm 46.0 \mathrm{sec}$ in patients with serum albumin $<2.85 \mathrm{~g} / \mathrm{dL}$. Additionally, Child-Pugh classification and serum chloride level were selected as the third and fourth splits, respectively.

Based on these results, we adopted serum albumin level as a biomarker to predict the impairment of cognitive function, determined as the prolongation of time required for the NCT-B in cirrhotic patients aged $\leq 65$ years.

\section{Characteristic features of patients aged $\leq 65$ years without PSS}

The presence of PSS often causes encephalopathy irrespectively of functional hepatic reserve. Thus, to accurately validate the predictive performance for cirrhosis-related cognitive dysfunction, patients with PSS were excluded from the 91 cirrhotic patients aged $\leq 65$ years. The demographic and clinical characteristics of the patients in the final analysis are presented in Table 1. Twenty-seven cirrhotic patients (16 men and 11 women; mean age, $56.7 \pm 8.6$ years) were included in the study. Among these subjects, $66.7 \%$ had an initial onset of $\mathrm{HE}, 33.3 \%$ had a recurrence of $\mathrm{HE}, 74.1 \%$ developed $\mathrm{HE}$ grade $\mathrm{I}$, $25.9 \%$ developed HE grade II, and the mean PSE index was 0.34 . The majority of patients $(70.4 \%)$ were classified as Child-Pugh B. The mean NCT-B was $92.2 \pm 45.2 \mathrm{sec}$, which was lower than that in cirrhotic patients aged $>66$ years $(133.1 \pm 43.1 \mathrm{sec})$, in agreement with the results of CART analysis (Figure 2 ). The mean blood ammonia concentration was $142.2 \mu \mathrm{g} / \mathrm{dL}$, and the mean BTR was 3.32 . 


Table 1. Characteristics of patients
\begin{tabular}{lc} 
Characteristics & 27 \\
\hline $\mathrm{N}$ & $56.7(8.6)$ \\
\hline Age (years old) & $16(59.3 \%) / 11(40.7 \%)$ \\
\hline Sex (male/female) & $64.6(11.6)$ \\
\hline Body weight (kg) & $18(66.7 \%) / 9(33.3 \%)$ \\
\hline Onset of HE (new-onset/recurrent) & $0.34(0.12)$ \\
\hline PSE index & $20(74.1 \%) / 7(25.9 \%)$ \\
\hline HE grade I/II & $3(11.1 \%) / 19(70.4 \%) / 5(18.5 \%)$ \\
\hline Child-Pugh classification $(\mathrm{A} / \mathrm{B} / \mathrm{C})$ & $142.2(41.1)$ \\
\hline Blood ammonia concentration $(\mu \mathrm{g} / \mathrm{dL})$ & \\
\hline
\end{tabular}

\section{Predictive performance of serum albumin level to identify cognitive dysfunction in cirrhotic patients}

To validate the predictive performance of serum albumin level in identifying the impairment of NCT-B time, we performed ROC curve analysis. In cirrhotic patients aged $\leq 65$ years without PSS, ROC curve analysis revealed that the optimal albumin level cutoff point was $3.05 \mathrm{~g} / \mathrm{dL}$ (sensitivity, $73.3 \%$; specificity, 91.7\%; PPV, 91.7\%; NPV, 73.3\%; accuracy, 81.5\%), and the area under the curve (AUC) value was 0.80 (95\% $\mathrm{Cl}, 0.60-0.98$ ) (Figure 3) for prolongation of NCT-B time. Among all cirrhotic patients, the AUC value to predict prolongation of NCT-B time was $0.63(95 \% \mathrm{Cl}, 0.54-0.71$ )(cutoff point, $3.15 \mathrm{~g} / \mathrm{dL}$; sensitivity, $61.2 \%$; specificity, $62.5 \%$ ), which was remarkably lower than that in patients aged $\leq 65$ years without PSS (Figure 3). These findings suggest that serum albumin level effectively identifies cognitive dysfunction in cirrhotic patients aged $\leq 65$ years without PSS.

\section{Efficiency of serum albumin level compared with other parameters related to cirrhosis-based HE}

Next, to evaluate the potential of other parameters related to the occurrence of cirrhosis-based HE to predict cognitive dysfunction, we performed similar analyses of BTR, serum ammonia level, PT-INR, and total bilirubin level. For detection of the prolongation of NCT-B time in cirrhotic patients aged $\leq 65$ years without PSS, the cutoff point was 3.295 for BTR (sensitivity, 46.7\%; specificity, 66.7\%; PPV, 63.6\%; NPV, $50.0 \%$; accuracy, 55.6\%), $121.8 \mu \mathrm{g} / \mathrm{dL}$ for serum ammonia level (sensitivity, $73.3 \%$; specificity, $58.3 \%$; PPV, 71.4\%; NPV, 61.5\%; accuracy, 66.7\%), 1.215 for PT-INR (sensitivity, 66.7\%; specificity, 66.7\%; PPV, 71.4\%; NPV, 61.5\%; accuracy, 66.7\%), and 0.850 for total bilirubin (sensitivity, 93.3\%; specificity, $41.7 \%$; PPV, 73.3\%; NPV, 66.7\%; accuracy, 70.4\%) (Supplementary Figure 1). The AUC value of serum albumin level (0.80) was higher than the AUC values of BTR $(0.46 ; 95 \% \mathrm{Cl}, 0.23-0.69)$, serum ammonia level $(0.61 ; 95 \%$ $\mathrm{Cl}, 0.38-0.83)$, PT-INR $(0.68 ; 95 \% \mathrm{Cl}, 0.46-0.87)$, and total bilirubin $(0.69 ; 95 \% \mathrm{Cl}, 0.46-0.89)$ (Figure 4), indicating that compared with other parameters, serum albumin level is distinctly capable of predicting impaired cognitive function in cirrhotic patients aged $\leq 65$ years.

\section{Discussion}

Definitive diagnosis of HE requires advanced clinical skills, because potential coexisting disorders must be considered, in addition to the underlying liver disease and complications of liver cirrhosis [1-3, 21, 22]. 
Previous clinical evidence showed that $54 \%$ of patients with cirrhosis and CHE or overt HE had comorbidities that could add to their symptoms in addition to HE, such as electrolyte imbalance, thiamine deficiency, sepsis, and cerebrovascular disorders [23-28]. Patients with overt HE show pathognomonic clinical symptoms, including asterixis, but these are not observed only in patients with HE. Such a backdrop has resulted in the development of various neuropsychological tests for the diagnosis of HE. These tests are clinically effective for detecting subclinical CHE. However, even in the case of CHE, individual differences in intelligence and cognitive abilities influence the results of these tests. Patients with greater intellectual abilities or cognitive reserves may achieve test results within the normal range even when they are experiencing increasing metabolic disequilibrium. On the other hand, these tests may be less accurate for diagnosing $\mathrm{CHE}$ in patients with lower cognitive ability. Variation in test results according to individual intelligence requires screening examination for parameters relevant to cirrhosis to select the patients who display cognitive dysfunction ascribed to liver cirrhosis.

Our CART analysis demonstrated that a lower level of serum albumin was the major clinical parameter related to liver cirrhosis that was associated with prolongation of the time required for the NCT-B in cirrhotic patients aged $\leq 65$ years. Remarkably, as confirmed by AUC, the predictive value of serum albumin level for detecting prolongation of the time required for the NCT-B was $80.0 \%$, which was higher than that of other cirrhosis-related parameters, including BTR, serum ammonia level, PT-INR, and serum bilirubin level. These results indicate that a lower albumin level may predict cognitive dysfunction in cirrhotic patients aged $\leq 65$ years without PSS. Human serum albumin (HSA) plays a pivotal role in the regulation of oncotic pressure and modulation of inflammatory pathways, as well as microvascular integrity [29]. Clinically, intravenous albumin supplementation has been utilized for treatment of patients with cirrhosis and ascites [30-32]. The rationale for the use of albumin is based on the recovery of effective circulating volume. Moreover, albumin binds many substances, such as nitric oxide (NO) and reactive oxygen species (ROS), that may be involved in the pathogenesis of both peripheral arterial vasodilatation and cardiac dysfunction in patients with cirrhosis and ascites [29, 33, 34]. Infusion of albumin can also be beneficial in patients with HE owing to its antioxidant properties and scavenging of ROS. Clinical studies have investigated the efficacy of administration of albumin in patients with HE. Jaran et al. showed that treatment with albumin produced a significant and sustained improvement of HE grade with declining plasma levels of malondialdehyde, a marker of ROS, in patients with alcoholic cirrhosis and diuretic-induced $\mathrm{HE}$, as compared with treatment with colloids [35]. Furthermore, Setoyama et al. demonstrated that BCAA supplementation could enhance the antioxidative potential by altering the redox state of HSA in the cirrhotic patients [36].

In addition to its antioxidant properties, an outstanding feature of HSA is its physiological ability to bind proinflammatory substances and inflammatory mediators [29,34]. Lipopolysaccharide (LPS) is a surface component of gram-negative and gram-positive bacteria that activates the innate immune system via tolllike receptor 4, and LPS-mediated cell activation requires a sequential interaction with several host proteins, including the LPS-binding protein CD14 and the coreceptor MD-2. In the pathogenesis of HE, microbe-derived LPS plays a potentially cardinal role in the development of systemic inflammation and neuroinflammation [37]. In patients with advanced cirrhosis, LPS increases the permeability of the blood- 
brain barrier and causes astrocyte swelling via production of $\mathrm{NO}$ and prostanoids in the brain microglia $[37,38]$. Clinical evidence suggests that endotoxemia is correlated with the severity of HE and the increased incidence of overt HE $[37,39]$. Noteworthy evidence is that HSA binds LPS by electrostatic and hydrophobic forces. Our previous study showed that the inactivation of plasma endotoxin was positively correlated with the endotoxin-binding capacity of plasma albumin in patients with Child-Pugh A and B cirrhosis [40]. An experiment with chronically ethanol-fed rats suggested that increased endotoxin-binding capacity of HSA served as a protective mechanism against the endotoxin [41]. In consideration of this evidence, the LPS-HSA complex could also play a role in the regulation of HE via the inflammatory response to bacterial infection in the cirrhotic state.

In summary, our results show that cirrhotic patients aged $\leq 65$ years with serum albumin levels $<$ $3.05 \mathrm{~g} / \mathrm{dL}$ are at high risk for HE-associated cognitive dysfunction and have to undergo neuropsychological tests. However, this study has several limitations that deserve consideration. First, lower albumin level had predictive potential for impaired cognition in cirrhotic patients aged $\leq 65$ years but was not effective in older patients. This finding indicates that using a single parameter of serum albumin level is inadequate to screen for HE-associated cognitive dysfunction in older cirrhotic patients. Second, this study utilized only the NCT-B, a convenient and common test, as a diagnostic method for $\mathrm{CHE}$. To validate the usefulness of serum albumin level in a screening examination for $\mathrm{CHE}$, the relation of CHE to the results of NCT-B and other neuropsychological tests should be elucidated.

\section{Conclusions}

To the best of our knowledge, this is the first study to identify lower serum albumin level as a clinical biomarker associated with impaired cognitive function in cirrhotic patients. We believe that this biomarker is clinically available for a screening examination to determine whether cirrhotic patients aged $\leq 65$ years without PSS should undergo neuropsychological tests for the diagnosis of early-stage HE.

\section{Abbreviations}

AST; aspartate aminotransferase, AUC; area under the curve, BCAA; branched-chain amino acid, BTR; branched-chain amino acids-to-tyrosine ratio, CART; classification and regression tree, CHE; covert hepatic encephalopathy, EDA; exploratory data analysis, GABA; $\gamma$-aminobutyric acid, HE; hepatic encephalopathy, HSA; human serum albumin, LPS; lipopolysaccharide, NCT; number connection test, NO; nitric oxide, NPV; negative predictive value, PPV; positive predictive value, PSE; portosystemic encephalopathy, PSS; portosystemic shunt, PT-INR; prothrombin time-international normalized ratio, ROC; receiver-operating characteristic, ROS; reactive oxygen species

\section{Declarations}

Ethics approval and consent to participate: The study was completed in compliance with Good Clinical Practice guidelines, and the ethical principles were based on the Declaration of Helsinki. The study 
protocols were approved by the Institutional Review Boards of the participating institutions, and the study was then carried out after obtaining written, informed consent from each patient according to the phase II/III, multicenter, randomized, evaluator-blinded, active-controlled trial and a phase III, multicenter, open trial [17].

Consent for publication: Not applicable.

Availability of data and materials: The datasets used and/or analyzed during the current study available from the corresponding author on reasonable request.

Competing Interests: Masaki Fujisawa and Ikuya Sato are employees of ASKA Pharmaceutical Co., Ltd. The other authors have no conflicts of interest.

Funding: This work does not have any funding supports.

Authors' contributions: K.K conducted data analysis and drafted the manuscript. M.F and I.S conducted data analysis. K.O and K.S offered the data support. H.Y designed and supervised the study. All authors read and gave final approval for the manuscript.

Acknowledgement: This study was conducted as a clinical trial by ASKA Pharmaceutical Co., Ltd.

\section{References}

1. Wijdicks EF. Hepatic Encephalopathy. N Engl J Med. 2016;375:1660-70.

2. Acharya C, Bajaj JS. Current Management of Hepatic Encephalopathy. Am J Gastroenterol. 2018;113:1600-12.

3. Amodio P. Hepatic encephalopathy: Diagnosis and management. Liver Int. 2018;38:966-75.

4. Bajaj JS, Wade JB, Sanyal AJ. Spectrum of neurocognitive impairment in cirrhosis: Implications for the assessment of hepatic encephalopathy. 2009;50:2014-21.

5. Bajaj JS, Cordoba J, Mullen KD, et al. Review article: the design of clinical trials in hepatic encephalopathy-an International Society for Hepatic Encephalopathy and Nitrogen Metabolism (ISHEN) consensus statement. Aliment Pharmacol Ther. 2011;33:739-47.

6. Shaw J, Bajaj JS. Covert Hepatic Encephalopathy: Can My Patient Drive? J Clin Gastroenterol. 2017;51:118-26.

7. Weissenborn K, Ennen JC, Schomerus H, et al. Neuropsychological characterization of hepatic encephalopathy. J Hepatol. 2001;34:768-73.

8. Mooney S, Hasssanein TI, Hilsabeck RC, et al. Utility of the repeatable battery for the assessment of neuropsychological status (RBANS) in patients with end-stage liver disease awaiting liver transplant. Arch Clin Neuropsychol. 2007;22:175-86.

9. Mardini H, Saxby BK, Record CO. Computerized psychometric testing in minimal encephalopathy and modulation by nitrogen challenge and liver transplant. 2008;135:1582-90. 
10. Bajaj JS, Hafeezullah M, Franco J, et al. Inhibitory control test for the diagnosis of minimal hepatic encephalopathy. 2008;135:1591-1600.e1.

11. Bajaj JS, Thacker LR, Heuman DM, et al. The stroop smartphone application is a short and valid method to screen for minimal hepatic encephalopathy. 2013;58:1122-32.

12. Badea MA, Drug VL, Dranga $M$, et al. Diagnosis of minimal hepatic encephalopathy in a tertiary care center from eastern Romania: validation of the psychometric hepatic encephalopathy score (PHES). Metab Brain 2016;31:1463-71.

13. Hoaglin D, John W. Tukey and data analysis. Stat Sci 2003;18:311-8.

14. Hendricks B, Mark-Carew M. Using exploratory data analysis to identify and predict patterns of human Lyme disease case clustering within a multistate region, 2010-2014. Spat Spatiotemporal Epidemiol. 2017;20:35-43.

15. Kawaguchi T, Inokuchi T, Honma T, et al. Factors associated with advanced hepatic fibrosis in patients with various internal diseases: a multicenter community-based survey. Hepatol Res. 2018;48:882-92.

16. Kawaguchi T, Tokushige $\mathrm{K}$, Hyogo $\mathrm{H}$, et al. A data mining-based prognostic algorithm for NAFLDrelated hepatoma patients: a nationwide study by the Japan Study Group of NAFLD. Sci Rep. 2018;8:10434.

17. Suzuki K, Endo R, Takikawa Y, et al. Efficacy and safety of rifaximin in Japanese patients with hepatic encephalopathy: A phase II/III, multicenter, randomized, evaluator-blinded, active-controlled trial and a phase III, multicenter, open trial. Hepatol Res. 2018;48:411-23.

18. Mochida S, Takikawa $Y$, Nakayama N, et al. Classification of the etiologies of acute liver failure in Japan: a report by the Intractable Hepato-Biliary Diseases Study Group of Japan. Hepatol Res. 2014;44:365-7.

19. Kawaguchi T, Konishi M, Kato A, et al. Updating the neuropsychological test system in Japan for the elderly and in a modern touch screen tablet society by resetting the cut-off values. Hepatol Res. 2017:47:1335-9.

20. Kanda Y. Investigation of the freely available easy-to-use software 'EZR' for medical statistics. Bone Marrow Transplant. 2013;48:452-58.

21. Caracciolo B, Xu W, Collins $S$, et al. Cognitive decline, dietary factors and gut-brain interactions. Mech Ageing Dev. 2014;136-137:59-69.

22. Bajaj JS, Ahluwalia V, Steinberg JL, et al. Elderly patients have an altered gut-brain axis regardless of the presence of cirrhosis. Sci Rep. 2016;6:38481.

23. Jiménez JV, Carrillo-Pérez DL, Rosado-Canto R, et al. Electrolyte and Acid-Base Disturbances in EndStage Liver Disease: A Physiopathological Approach. Dig Dis Sci. 2017;62:1855-71.

24. Haberl J, Zollner G, Fickert P, et al. To salt or not to salt?-That is the question in cirrhosis. Liver Int. 2018;38:1148-59. 
25. Lévy S, Hervé C, Delacoux E, et al. Thiamine deficiency in hepatitis $C$ virus and alcohol-related liver diseases. Dig Dis Sci. 2002;47:543-8.

26. Sureka B, Bansal K, Patidar Y, et al. Neurologic Manifestations of Chronic Liver Disease and Liver Cirrhosis. Curr Probl Diagn Radiol. 2015;44:449-61.

27. Gustot T, Fernandez J, Szabo G, et al. Sepsis in alcohol-related liver disease. J Hepatol. 2017;67:1031-50.

28. Xiong J, Xu W, Huang H, Bian J, et al. Cirrhosis and risk of stroke: A systematic review and metaanalysis. Atherosclerosis. 2018;275:296-303.

29. Arroyo V, García-Martinez R, Salvatella X. Human serum albumin, systemic inflammation, and cirrhosis. J Hepatol. 2014;61:396-407.

30. Gentilini P, Casini-Raggi V, Di Fiore G, et al. Albumin improves the response to diuretics in patients with cirrhosis and ascites: results of a randomized, controlled trial. J Hepatol. ;30:639-45.

31. Bernardi $M$, Caraceni $P$, Navickis RJ, et al. Albumin infusion in patients undergoing large-volume paracentesis: a meta-analysis of randomized trials. Hepatology. 2012;55:1172-81.

32. Fukui H, Saito H, Ueno Y, et al. Evidence-based clinical practice guidelines for liver cirrhosis 2015. J Gastroenterol. 2016;51:629-50.

33. Taverna M, Marie AL, Mira JP, et al. Specific antioxidant properties of human serum albumin. Ann Intensive Care. 2013;3:4.

34. Garcia-Martinez R, Caraceni P, Bernardi M, et al. Albumin: pathophysiologic basis of its role in the treatment of cirrhosis and its complications. 2013;58:1836-46.

35. Jalan R, Kapoor D. Reversal of diuretic-induced hepatic encephalopathy with infusion of albumin but not colloid. Clin Sci (Lond). 2004;106:467-74.

36. Setoyama $\mathrm{H}$, Tanaka $\mathrm{M}$, Nagumo K, et al. Oral branched-chain amino acid granules improve structure and function of human serum albumin in cirrhotic patients. J Gastroenterol. 2017;52:754-65.

37. Jayakumar AR, Rama Rao KV, Norenberg MD. Neuroinflammation in hepatic encephalopathy: mechanistic aspects. J Clin Exp Hepatol. 2015;5:S21-S28.

38. Verma S, Nakaoke R, Dohgu S, et al. Release of cytokines by brain endothelial cells: A polarized response to lipopolysaccharide. Brain Behav Immun. 2006;20:449-55.

39. Ahluwalia V, Betrapally NS, Hylemon PB, et al. Impaired Gut-Liver-Brain Axis in Patients with Cirrhosis. Sci Rep. 2016;6:26800.

40. Fukui $\mathrm{H}$, Tsujita $\mathrm{S}$, Matsumoto $\mathrm{M}$, et al. Endotoxin inactivating action of plasma in patients with liver cirrhosis. Liver. 1995;15:104-9.

41. Kitano H, Fukui H, Okamoto $\mathrm{Y}$, et al. Role of albumin and high-density lipoprotein as endotoxinbinding proteins in rats with acute and chronic alcohol loading. Alcohol Clin Exp Res. 1996;20:73A76A.

\section{Figures}




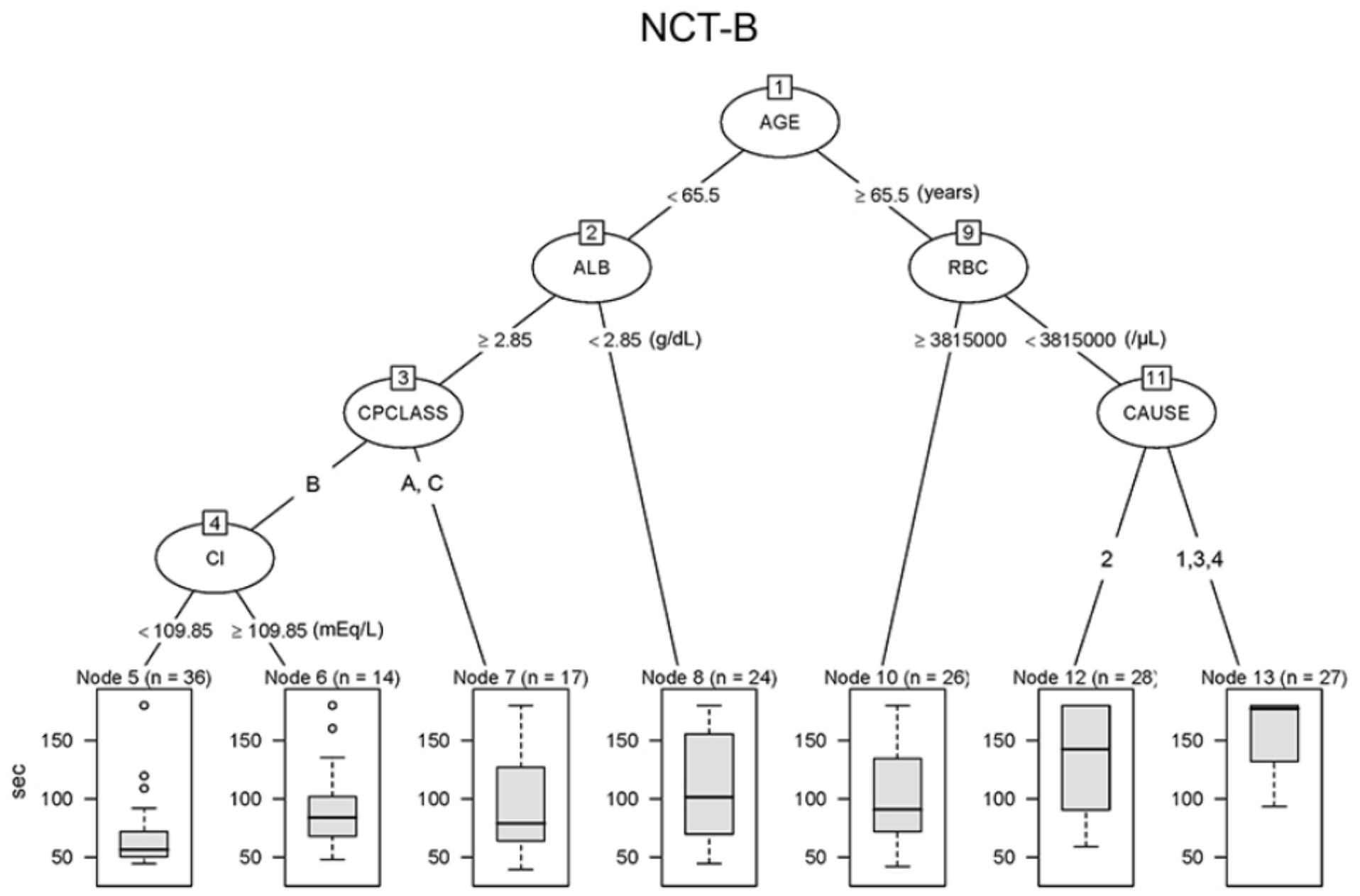

Figure 1

Classification and regression tree for clinical profiles. Clinical parameters were associated with number connection test-B (NCT-B) in 172 Japanese patients with hepatic encephalopathy. ALB; serum albumin level, CPCLASS; child-pugh classification, $\mathrm{Cl}$; serum chroride level, RBC; red blood cell count, CAUSE; etiology of liver cirrhosis: 1; hepatitis B, 2; hepatitis C, 3; alcoholic liver injury, 4; the others. 


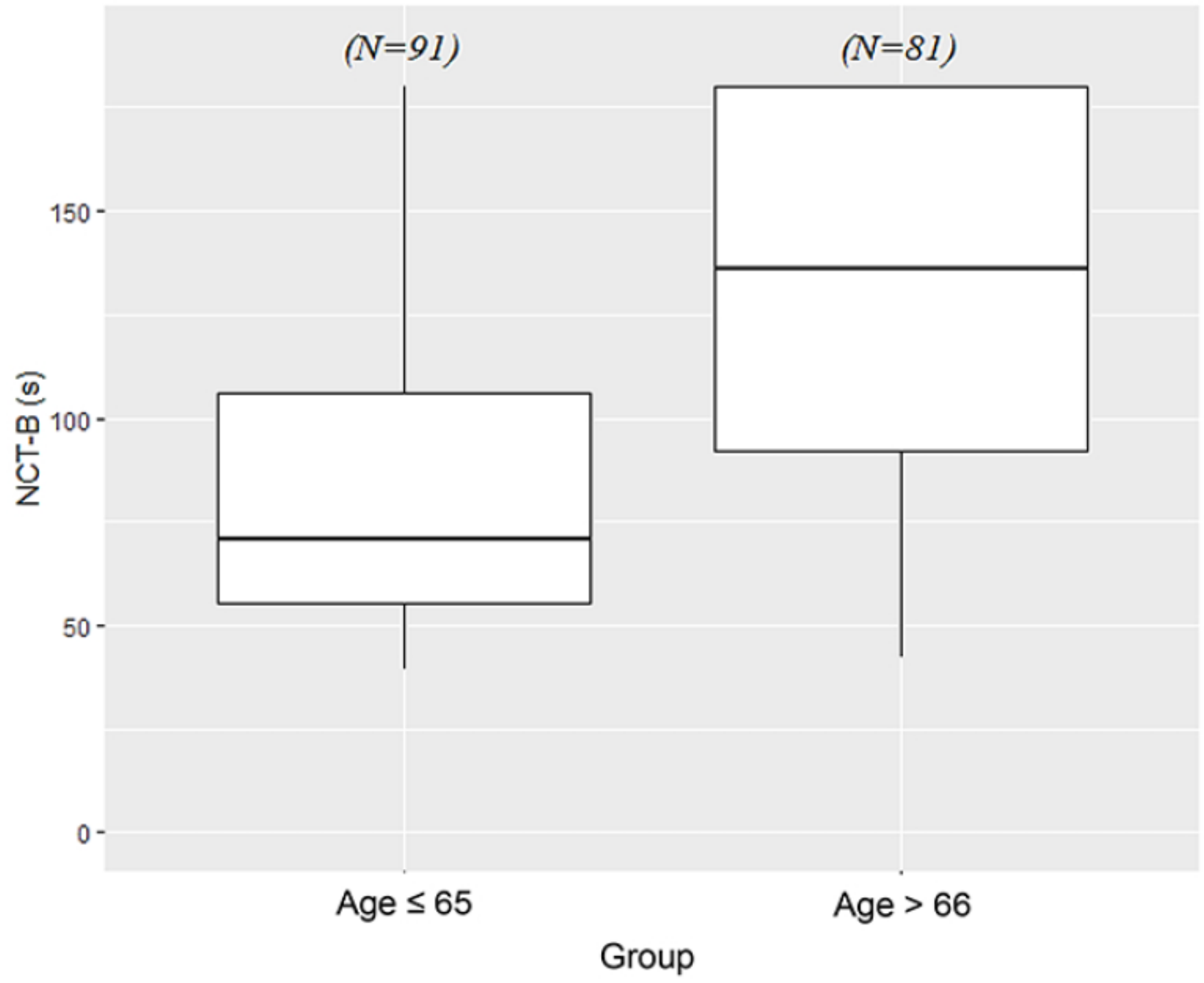

Figure 2

The mean time for number connection test (NCT-B). Comparison the groups of patients between aged $\leq$ 65 years $(\mathrm{N}=91)$ and $>66$ years $(\mathrm{N}=81)$. 
ROC curvelAlbumin

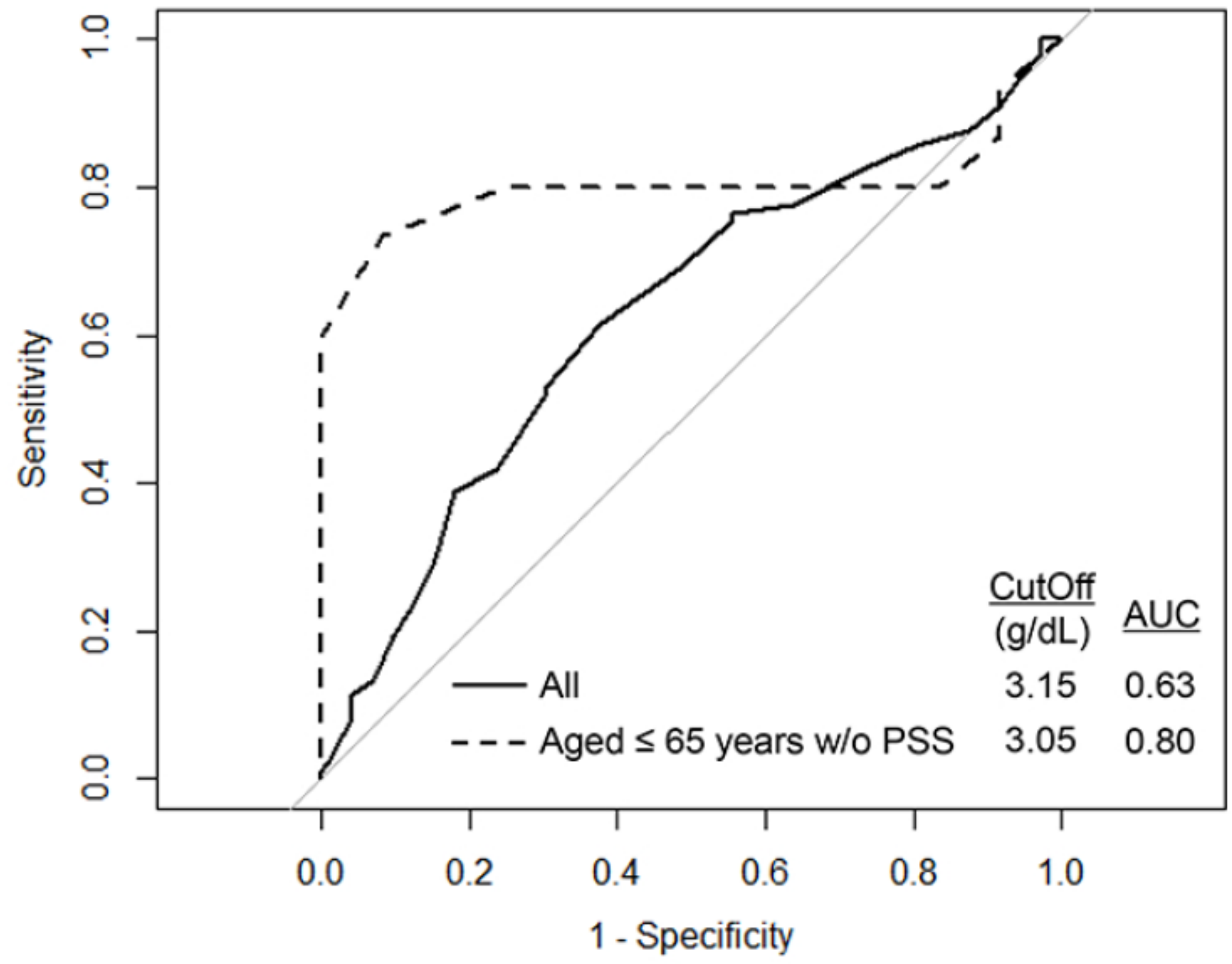

Figure 3

Receiver operating characteristic curve analysis and area under curve (AUC) value. Data analysis for predictive performance of serum albumin level for impairment of number connection test-B. W/o PSS; without portosystemic shunt 
ROC curve

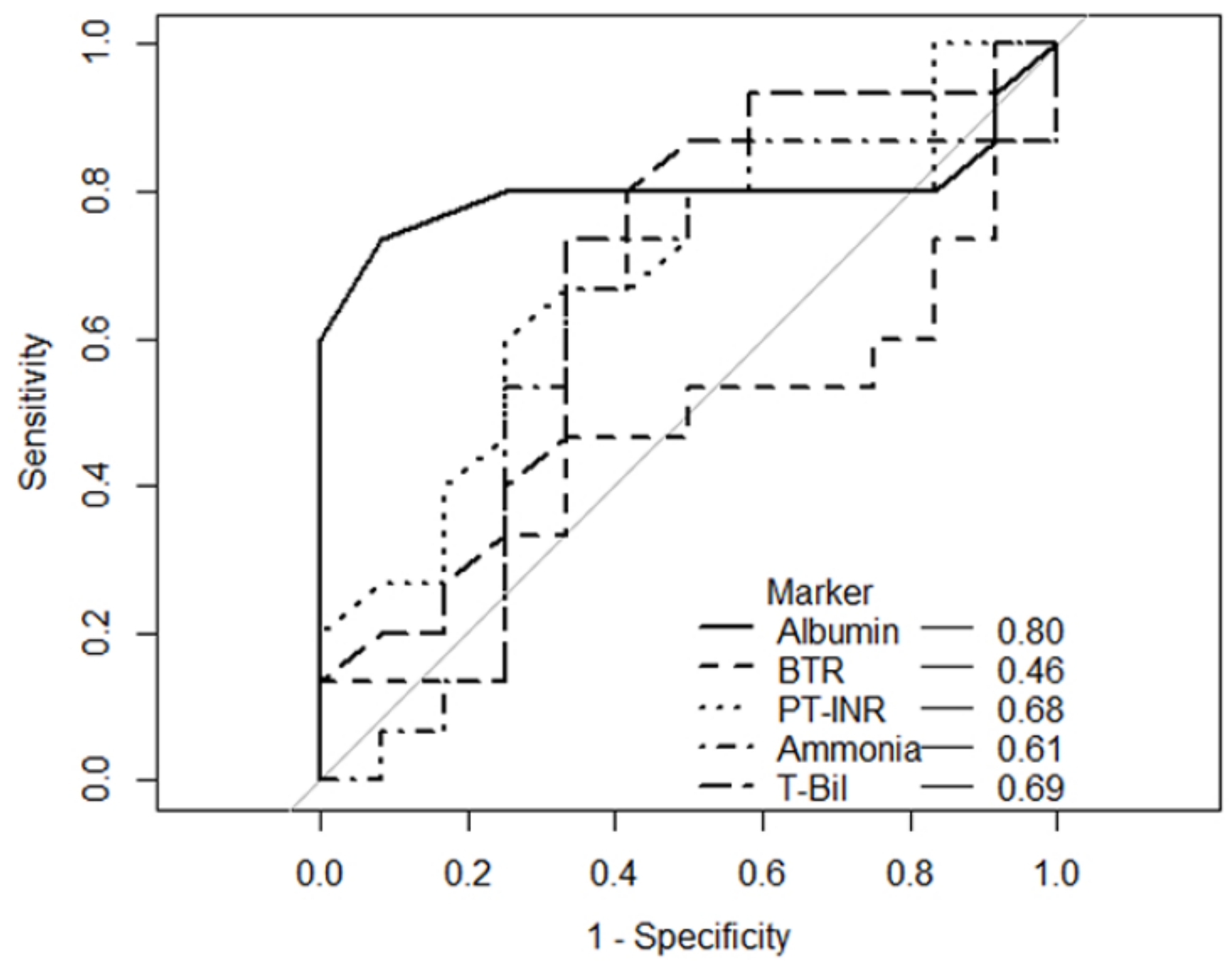

Figure 4

Comparison among cirrhosis-related parameters in predictive performance for impairment of number connection test-B. Data analysis for cirrhotic patients aged $\leq 65$ years without portosystemic shunt. Albumin; serum albumin level, BTR; branched-chain amino acids-to-tyrosine ratio, PT-INR; prothrombin time-international normalized ratio, Ammonia; serum ammonia level, T-bil; total bilirubin level.

\section{Supplementary Files}

This is a list of supplementary files associated with this preprint. Click to download.

- Supplementaryfigurelegends.docx

- Listofethicalcommittees.docx 
- Supplementaryfigure1.pdf

Page 16/16 